Proc Indian Natn Sci Acad 81 No. 1 February 2015 Special Issue, pp. 75-85

(C) Printed in India.

DOI: 10.16943/ptinsa/2015/v81i1/48054

\title{
Imaginary Part of the Medium Modified Heavy Quark Potential LATA THAKUR ${ }^{\star}$, UTTAM KAKADE and BINOY KRISHNA PATRA \\ Department of Physics, Indian Institute of Technology Roorkee 247 667, India
}

(Received on 31 May 2014; Accepted on 25 June 2014)

\begin{abstract}
We have calculated the dissociation of quarkonia through an imaginary potential which is obtained by correcting both the perturbative and non-perturbative terms of the potential at $\mathrm{T}=0$ through the dielectric function in real-time formalism. The real-part of the potential becomes stronger and thus makes the quarkonia more bound whereas the (magnitude) imaginary-part too becomes larger and thus contribute more to the thermal width, compared to the medium-contribution of the Coulomb term alone. We have also extended our calculation to anisotropic medium, by calculating the leading anisotropic corrections to the propagators in Keldysh representation. The presence of anisotropy makes the real-part of the potential stronger but the imaginary-part is weakened slightly and the competition between them results in higher dissociation temperatures compared to isotropic medium.
\end{abstract}

Key Words : Quantum Chromodynamics; Debye Mass; Momentum Anisotropy; String Tension; Dielectric Permittivity; Heavy Quark Potential; Decay Width

\section{Introduction}

Heavy quarkonium systems have turned out to provide extremely useful probes for the deconfined state of matter because the force between a heavy quark and its anti-quark, is weakened due to the presence of light quarks and gluons which leads to the dissociation of quarkonium bound states (Matsui and Satz, 1986). The medium effects can be envisaged through a temperature-dependent heavy quark potentials and have been studied over the decades either phenomenologically or through lattice based free-energy calculations (Mocsy and Petreczky, 2008). Among the recent theoretical developments in the quarkonium studies, the first-principle calculations of imaginary contributions to the heavy quark potential either due to gluonic Landau damping (Laine et al., 2007) or due to the singlet to octet transitions etc. (Brambilla et al., 2008), which describe the decaying of the $Q \bar{Q}$ correlation with its initial state due to scatterings in the plasma

^Author for Correspondence : E-mail: thakurphyom@gmail.com 
(Beraudo et al., 2008), are well known. Earlier it was thought that a quarkonium state is dissociated when the screening becomes so strong that it inhibits the formation of bound states but nowadays a quarkonium is dissociated at a lower temperature (Laine et al., 2007; Burnier et al., 2008) even though its binding energy is nonvanishing, rather is overtaken by the Landau-damping induced thermal width (Laine et al., 2007; Hatsuda, 2013). Although the lattice studies predicts a sizable imaginary component in the potential (Rothkopf et al., 2009, 2012), it may not be reliable because the necessary quality of the data has not yet been achieved. One thus needs inadvertent support from the potential models at finite temperature as an important tool to complement the lattice studies. Since the string-tension does not vanish abruptly at the deconfinement point (Cheng et al., 2008), one should study its effects on heavy quark potential even above $T_{c}$. So we aim here to calculate the imaginary part, in addition to the real part of the potential both in isotropic and anisotropic medium by correcting the full Cornell potential and not its Coulomb part alone.

The structure of this paper is as follows: We have reviewed the potential introduced in (Agotiya et al., 2009) and extended it to the imaginary part of the potential for both the isotropic as well as anisotropic medium. We have started with propagators and self energies in Keldysh representation and their evaluation in HTL resummed theory. Then we have studied the dissociation of charmonium and bottomonium states by calculating their (thermal) widths and binding energies. Finally we conclude our main results.

\section{Potential in a Hot QCD Medium}

The medium-modification to the vacuum potential can be obtained by correcting its both short and longdistance part with a dielectric function $\epsilon(p)$ encoding the effect of deconfinement (Agotiya et al., 2009). Fourier transform of potential at vanishing frequency gives the desired non-relativistic potential at finite temperature.

$$
V(r, T)=\int \frac{d^{3} \mathbf{p}}{(2 \pi)^{3 / 2}}\left(e^{i \mathbf{p} \cdot \mathbf{r}}-1\right) \frac{V(p)}{\epsilon(p)},
$$

where the $r$-independent term needed to renormalize the heavy quark free energy is the perturbative free energy of quarkonium at infinite separation (Dumitru et al., 2009). $V(p)$ is the Fourier transform of the potential given by:

$$
V(p)=-\sqrt{(2 / \pi)} \frac{\alpha}{p^{2}}-\frac{4 \sigma}{\sqrt{2 \pi} p^{4}} .
$$

$\epsilon(p)$ is the dielectric permittivity given by (Schneider, 2002):

$$
\epsilon(p)=\left(1+\frac{\Pi_{L}(0, p, T)}{p^{2}}\right) \equiv\left(1+\frac{m_{D}^{2}}{p^{2}}\right),
$$

where $m_{D}$ is the screening mass. 
However in the presence of non-perturbative effects, the dependence of the dielectric function on the Debye mass may get modified. In this work the same (perturbative) screening scale are employed for both the linear and Coulombic terms which may not look plausible. It would be interesting to see the effects of different scales for the Coulomb and linear pieces of the $\mathrm{T}=0$ potential in Ref. (Megias et al., 2007,2011 ) rather than a single one, where the non-perturbative effects have been incorporated beyond the deconfinement temperature through dimension-two gluon condensates. The difference with their calculation lies in the large distance limit of the potential and is found more attractive than our potential.

The dielectric permittivity can be calculated once the self energies and propagators are obtained in HTL resummation theory in the real-time formalism (Carrington et al., 1999). The gluon self-energy can be obtained by folding the approximated phase-space distribution in anisotropic medium (Romatschke and Strickland, 2003) $(\xi \ll 1)$ as:

$$
f_{\text {aniso }}(\mathbf{k})=\mathbf{f}_{\text {iso }}\left(\sqrt{\mathbf{k}^{2}+\xi(\mathbf{k} \cdot \mathbf{n})^{2}}\right)
$$

and hence the resummed propagator. The contribution from the quark loop (Dumitru et al., 2009) to the gluon self energy with external and internal momenta as $P\left(p_{0}, \mathbf{p}\right)$ and $K\left(k_{0}, \mathbf{k}\right)$, respectively (with $Q=$ $K-P)$ :

$$
\Pi^{\mu \nu}(P)=-\frac{i}{2} N_{f} g^{2} \int \frac{d^{4} K}{(2 \pi)^{4}} \operatorname{tr}\left[\gamma^{\mu} S(Q) \gamma^{\nu} S(K)\right]
$$

In HTL-limit, the quark and gluon loops together give the isotropic part of retarded (advanced) selfenergy (Dumitru, 2009)

$$
\Pi_{R, A(i s o)}^{L}(P)=m_{D}^{2}\left(\frac{p_{0}}{2 p} \ln \frac{p_{0}+p \pm i \epsilon}{p_{0}-p \pm i \epsilon}-1\right),
$$

with the prescriptions $+i \epsilon(-i \epsilon)$, for the retarded (advanced) self-energies, respectively whereas the anisotropic part for the retarded (advanced) self energies are

$$
\begin{aligned}
\Pi_{R, A(\text { aniso })}^{L}(P) & =\frac{m_{D}^{2}}{6}\left(1+\frac{3}{2} \cos 2 \theta_{p}\right)+\Pi_{R(\text { iso })}^{L}(P) \\
& \times\left(\cos \left(2 \theta_{p}\right)-\frac{p_{0}^{2}}{2 p^{2}}\left(1+3 \cos 2 \theta_{p}\right)\right) .
\end{aligned}
$$

Similarly the isotropic and anisotropic terms for the temporal component of the symmetric part are given by

$$
\begin{aligned}
& \Pi_{F(\text { iso })}^{L}(P)=-2 \pi i m_{D}^{2} \frac{T}{p} \Theta\left(p^{2}-p_{0}^{2}\right) \\
& \Pi_{F(\text { aniso })}^{L}(P)=\frac{3}{2} \pi i m_{D}^{2} \frac{T}{p}\left(\sin ^{2} \theta_{p}+\frac{p_{0}^{2}}{p^{2}}\left(3 \cos ^{2} \theta_{p}-1\right)\right) \Theta\left(p^{2}-p_{0}^{2}\right) .
\end{aligned}
$$


Thus the gluon self-energy is found to have both real and imaginary part which are responsible for the Debye screening and the Landau damping, respectively. The real part of the static potential can be obtained from the temporal component of retarded (or advanced) propagator (in static limit)

$$
\operatorname{Re} D_{R, A}^{00}(0, p)=-\frac{1}{\left(p^{2}+m_{D}^{2}\right)}+\xi \frac{m_{D}^{2}}{6\left(p^{2}+m_{D}^{2}\right)^{2}}\left(3 \cos 2 \theta_{p}-1\right)
$$

while for the imaginary part of the potential, the imaginary part of the temporal component of symmetric propagator is given by

$$
\begin{aligned}
\operatorname{Im} D_{F}^{00}(0, p) & =\frac{-2 \pi T m_{D}^{2}}{p\left(p^{2}+m_{D}^{2}\right)^{2}}+\xi\left(\frac{3 \pi T m_{D}^{2}}{2 p\left(p^{2}+m_{D}^{2}\right)^{2}} \sin ^{2} \theta_{p}\right. \\
& \left.-\frac{4 \pi T m_{D}^{4}}{p\left(p^{2}+m_{D}^{2}\right)^{3}}\left(\sin ^{2} \theta_{p}-\frac{1}{3}\right)\right) .
\end{aligned}
$$

\section{Real Part of the Potential}

The real part of the static potential can thus be obtained from eq. (1) by substituting the dielectric permittivity $\epsilon(p)$ in terms of the physical " 11 "- component of the gluon propagator. The relation between the dielectric permittivity and the static limit of the "00"-component of gluon propagator in Coulomb gauge is obtained

from the linear response theory (Kapusta and Gale, 1996): $\epsilon^{-1}(p)=-\lim _{\omega \rightarrow 0} p^{2} D_{11}^{00}(\omega, p)$, where the real and imaginary parts of $D_{11}^{00}$ can be written as

$$
R e D_{11}^{00}(\omega, p)=\frac{1}{2}\left(D_{R}^{00}+D_{A}^{00}\right) \text { and } \operatorname{Im} D_{11}^{00}(\omega, p)=\frac{1}{2} D_{F}^{00}
$$

The real-part of the potential is then obtained as

$$
\begin{aligned}
R e V_{(\text {aniso })}(\mathbf{r}, \xi, T)= & \int \frac{d^{3} \mathbf{p}}{(2 \pi)^{3 / 2}}\left(e^{i \mathbf{p} \cdot \mathbf{r}}-1\right)\left(-\sqrt{(2 / \pi)} \frac{\alpha}{p^{2}}-\frac{4 \sigma}{\sqrt{2 \pi} p^{4}}\right) \times \\
& p^{2}\left[\frac{1}{\left(p^{2}+m_{D}^{2}\right)}-\frac{\xi m_{D}^{2}}{6\left(p^{2}+m_{D}^{2}\right)^{2}}\left(3 \cos \left(2 \theta_{p}\right)-1\right)\right],
\end{aligned}
$$

where $\theta_{p}$ is the angle between $\mathbf{r}$ and $\mathbf{n}$ (direction of anisotropy). After performing the integration, the 
real-part of the potential in anisotropic medium becomes (Thakur et al., 2013)

$$
\begin{aligned}
\Re V_{\text {aniso }}\left(r, \theta_{r}, T\right) & =\frac{2 \sigma}{m_{D}}\left(\frac{e^{-\hat{r}}-1}{\hat{r}}+1\right)-\alpha m_{D}\left(\frac{e^{-\hat{r}}}{\hat{r}}+1\right)+\xi \frac{e^{-\hat{r}}}{\hat{r}} \\
& \times\left[\frac{2 \sigma}{m_{D}}\left(\frac{e^{\hat{r}}-1}{\hat{r}^{2}}+\frac{\hat{r}^{2} e^{\hat{r}}-3}{3 \hat{r}}-\frac{5 e^{\hat{r}}-\hat{r}+1}{12}\right)\right. \\
& -\frac{\alpha m_{D}}{2}\left(\frac{e^{\hat{r}}-1}{\hat{r}^{2}}-\frac{1}{\hat{r}}-\frac{2 \hat{r} e^{\hat{r}}-\hat{r}+3}{6}\right) \\
& +\left[\frac{2 \sigma}{m_{D}}\left(3 \frac{e^{\hat{r}}-1}{\hat{r}^{2}}-\frac{3}{\hat{r}}-\frac{e^{\hat{r}}+\hat{r}+5}{4}\right)\right. \\
& \left.\left.-\frac{\alpha m_{D}}{2}\left(3 \frac{e^{\hat{r}}-1}{\hat{r}^{2}}-\frac{3}{\hat{r}}-\frac{\hat{r}+3}{2}\right)\right] \cos 2 \theta_{r}\right] \\
& =\Re V_{\text {iso }}(r, T)+V_{\text {tensor }}\left(r, \theta_{r}, T\right) .
\end{aligned}
$$

Thus the anisotropy in the momentum space introduces an angular $\left(\theta_{r}\right)$ dependence, in addition to the inter-particle separation $(r)$, to the real part of the potential. The real potential becomes stronger with the increase of anisotropy because the (anisotropic) Debye mass $m_{D}(\xi, T)$ (or equivalently angular-dependent Debye mass $m_{D}\left(\theta_{r}, T\right)$ ) in an anisotropic medium is always smaller than in an isotropic medium.

\section{Imaginary Part of the Potential: Thermal Width, $\Gamma$}

The imaginary part of the potential plays an important role in weakening the bound state peak or transforming it to mere threshold enhancement. It leads to a finite width $(\Gamma)$ for the resonance peak in the spectral function, which, in turn, determines the dissociation temperature. In recent years the imaginary part with a momentum-space anisotropy and its effects on the thermal widths of the resonance states have been studied (Dumitru et al., 2009; Margotta et al., 2011; Dumitru, 2011), with the medium-modification to the perturbative (Coulomb) term only. We follow their work by including the medium corrections to both perturbative (Coulombic) and non-perturbative (string) terms in a weakly anisotropic medium by the imaginary part of the dielectric function:

$$
\begin{aligned}
\operatorname{Im} V_{\text {(aniso) }}(\mathbf{r}, \xi, T) & =-\int \frac{d^{3} \mathbf{p}}{(2 \pi)^{3 / 2}}\left(e^{i \mathbf{p} \cdot \mathbf{r}}-1\right)\left(-\sqrt{\frac{2}{\pi}} \frac{\alpha}{p^{2}}-\frac{4 \sigma}{\sqrt{2 \pi} p^{4}}\right) p^{2} \\
& \times\left[\frac{-\pi T m_{D}^{2}}{p\left(p^{2}+m_{D}^{2}\right)^{2}}+\xi\left[\frac{3 \pi T m_{D}^{2}}{4 p\left(p^{2}+m_{D}^{2}\right)^{2}} \sin ^{2} \theta_{p}\right.\right. \\
& \left.-\frac{2 \pi T m_{D}^{4}}{p\left(p^{2}+m_{D}^{2}\right)^{3}}\left(\sin ^{2} \theta_{p}-\frac{1}{3}\right)\right] \\
& \equiv \operatorname{ImV}_{1(\text { aniso })}(\mathbf{r}, \xi, T)+\operatorname{ImV}_{2(\text { aniso })}(\mathbf{r}, \xi, T)
\end{aligned}
$$

where $\operatorname{Im} V_{1(\text { aniso) }}(\mathbf{r}, \xi, T)$ and $\operatorname{Im} V_{2(\text { aniso })}(\mathbf{r}, \xi, T)$ are the imaginary contributions corresponding to the Coulombic and linear terms in anisotropic medium, respectively. The contribution due to the perturbative 
term in the leading-order is given by (Dumitru et al., 2009)

$$
\operatorname{Im} V_{1(\text { aniso })}(\mathbf{r}, \xi, T)=-\alpha T\left(\phi_{0}(\hat{r})+\xi\left[\phi_{1}\left(\hat{r}, \theta_{r}\right)+\phi_{2}\left(\hat{r}, \theta_{r}\right)\right]\right)
$$

where the functions $\phi_{0}(\hat{r}), \phi_{1}\left(\hat{r}, \theta_{r}\right)$ and $\phi_{2}\left(\hat{r}, \theta_{r}\right)$ are given by

$$
\begin{aligned}
\phi_{0}(\hat{r}) & =-\alpha T\left(-\frac{\hat{r}^{2}}{9}\left(-4+3 \gamma_{E}+3 \log \hat{r}\right)\right) \\
\phi_{1}\left(\hat{r}, \theta_{r}\right) & =\frac{\hat{r}^{2}}{600}\left[123-90 \gamma_{E}-90 \log \hat{r}+\cos 2 \theta_{r}\left(-31+30 \gamma_{E}+30 \log \hat{r}\right)\right] \\
\phi_{2}\left(\hat{r}, \theta_{r}\right) & =\frac{\hat{r}^{2}}{90}\left(-4+3 \cos 2 \theta_{r}\right)
\end{aligned}
$$
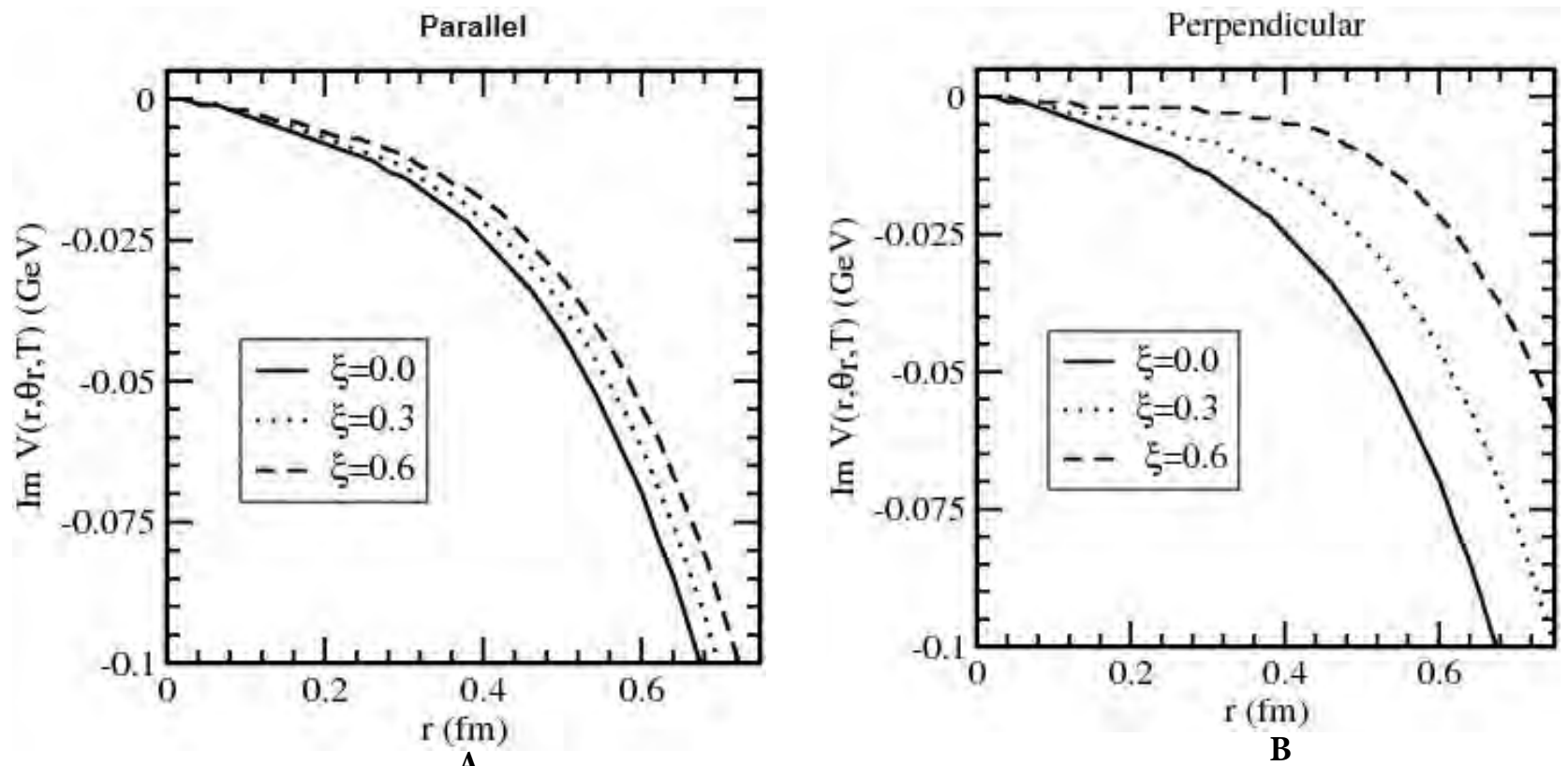

A

B

Fig. 1: Imaginary part of the potential for parallel (A) and perpendicular (B) alignment in an anisotropic medium

Similarly the imaginary part due to the non-perturbative (linear) term has also the isotropic and anisotropic term:

$$
\operatorname{Im} V_{2(\text { aniso })}(r, \xi, T)=\frac{2 \sigma T}{m_{D}^{2}}\left(\psi_{0}(\hat{r})-\xi\left[\psi_{1}\left(\hat{r}, \theta_{r}\right)+\psi_{2}\left(\hat{r}, \theta_{r}\right)\right]\right)
$$


where the functions $\psi_{0}(\hat{r}), \psi_{1}\left(\hat{r}, \theta_{r}\right)$ and $\psi_{2}\left(\hat{r}, \theta_{r}\right)$ are given by

$$
\begin{aligned}
\psi_{0}(\hat{r}) & =\frac{\hat{r}^{2}}{6}+\left(\frac{-107+60 \gamma_{E}+60 \log (\hat{r})}{3600}\right) \hat{r}^{4}+O\left(\hat{r}^{5}\right), \\
\psi_{1}\left(\hat{r}, \theta_{r}\right) & =\frac{\hat{r}^{2}}{10}+\frac{\left(-739+420 \gamma_{E}+420 \log (\hat{r})\right) \hat{r}^{4}}{39200} \\
& +\left(-\frac{\hat{r}^{2}}{20}+\frac{\left(176-105 \gamma_{E}-105 \log (\hat{r})\right) \hat{r}^{4}}{14700}\right) \cos ^{2} \theta_{r}, \\
\psi_{2}\left(\hat{r}, \theta_{r}\right) & =-\frac{4}{3}\left[\frac{7 \hat{r}^{2}}{120}-\frac{11 \hat{r}^{4}}{3360}+O\left(\hat{r}^{5}\right)\right] \\
& -4\left[-\frac{\hat{r}^{2}}{60}+\frac{\hat{r}^{4}}{840}+O\left(\hat{r}^{5}\right)\right] \cos ^{2} \theta_{r},
\end{aligned}
$$

respectively and $\gamma_{E}$ is the Euler-Gamma constant. Finally the short and long-distance contributions, in the leading logarithmic order, gives the imaginary part of the potential in the anisotropic medium
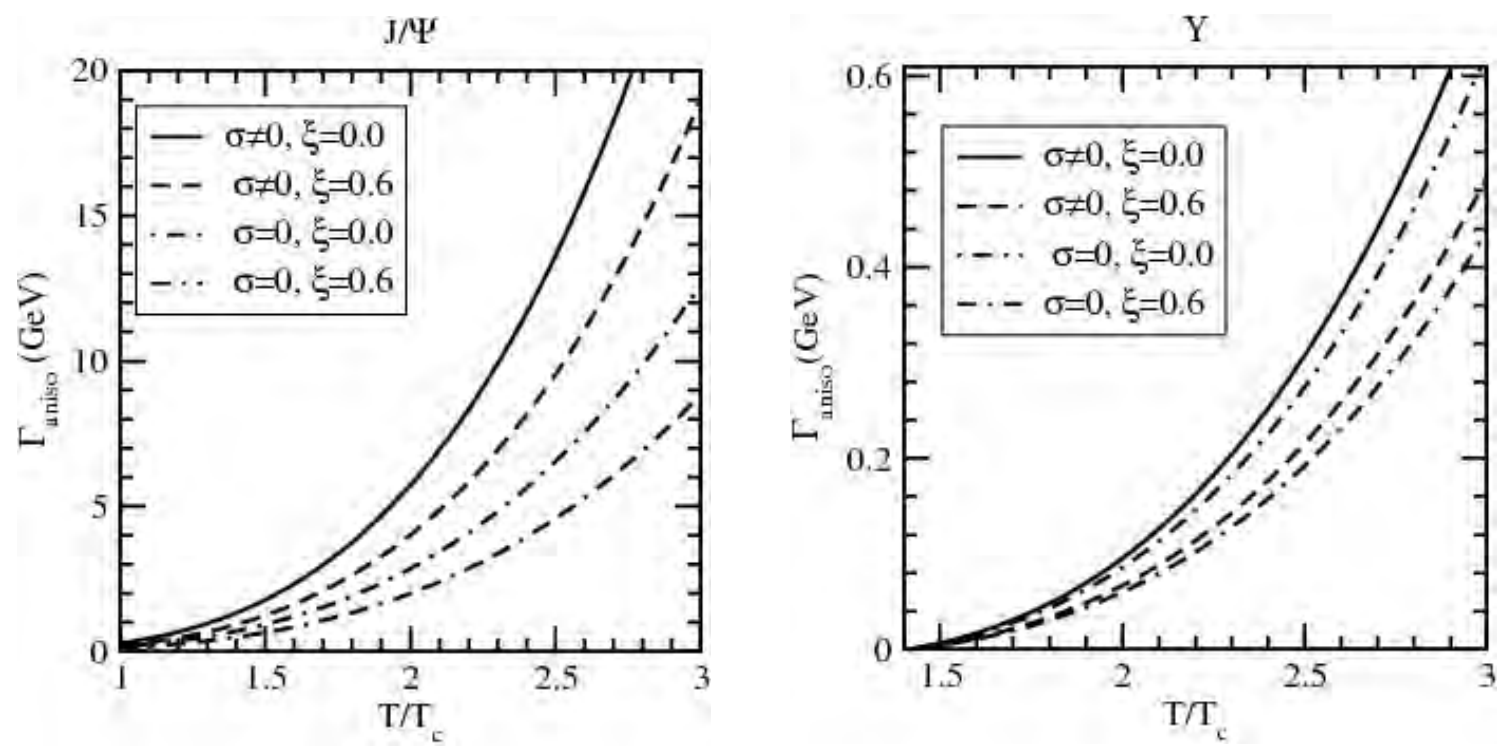

Fig. 2: The thermal width for the $J / \psi$ and $\Upsilon$ states in the anisotropic medium

$$
\begin{aligned}
\operatorname{Im} V_{(\text {aniso })}\left(r, \theta_{r}, T\right)= & -T\left(\frac{\alpha \hat{r}^{2}}{3}+\frac{\sigma \hat{r}^{4}}{30 m_{D}^{2}}\right) \log \left(\frac{1}{\hat{r}}\right) \\
& +\xi T\left[\left(\frac{\alpha \hat{r}^{2}}{5}+\frac{3 \sigma \hat{r}^{4}}{140 m_{D}^{2}}\right)\right. \\
& \left.-\cos ^{2} \theta_{r}\left(\frac{\alpha \hat{r}^{2}}{10}+\frac{\sigma \hat{r}^{4}}{70 m_{D}^{2}}\right)\right] \log \left(\frac{1}{\hat{r}}\right),
\end{aligned}
$$


which is found to be smaller than the isotropic medium and decreases with the increase of anisotropy (shown in Fig. 1). The imaginary part of the potential, in small-distance limit, is a perturbation to the vacuum potential and thus provides an estimate for the width $(\Gamma)$ of a resonance state and can be calculated, in a first-order perturbation, by folding with the unperturbed (1S) Coulomb wave function

$$
\begin{aligned}
\Gamma_{\text {(aniso) }}= & \int d^{3} \mathbf{r}|\Psi(r)|^{2}\left[\alpha T \hat{r}^{2} \log \left(\frac{1}{\hat{r}}\right)\left(\frac{1}{3}-\xi \frac{3-\cos 2 \theta_{r}}{20}\right)\right. \\
& \left.+\frac{2 \sigma T}{m_{D}^{2}} \hat{r}^{4} \log \left(\frac{1}{\hat{r}}\right) \frac{1}{20}\left(\frac{1}{3}-\xi \frac{2-\cos 2 \theta_{r}}{14}\right)\right] \\
= & T\left(\frac{4}{\alpha m_{Q}^{2}}+\frac{12 \sigma}{\alpha^{2} m_{Q}^{4}}\right)\left(1-\frac{\xi}{2}\right) m_{D}^{2} \log \frac{\alpha m_{Q}}{2 m_{D}} .
\end{aligned}
$$

From the (Fig. 2) it is clear that the width always increases with the temperature. The non-perturbative string term, in addition to the Coulomb term, makes the width larger than the earlier result with the perturbative Coulomb term (Dumitru, 2011) only and thus the damping of the exchanged gluon in the heat bath provides larger contribution to the dissociation rate. Width becomes smaller in anisotropic medium than in isotropic medium and gets narrower with the increase of anisotropy because $\Gamma$ is approximately proportional to the (square) Debye mass and the Debye mass decreases in the anisotropic medium.

\section{Real and Imaginary Binding Energies: Dissociation Temperatures}

In order to understand the in-medium properties of the quarkonium states, one need to solve the Schrödinger equation with both the real and imaginary part of the finite temperature potential. The real part of binding energy may be obtained from the radial part of the Schrödinger equation (of the isotropic component) plus the first-order perturbation due to the anisotropic component as

$$
\operatorname{Re} E_{\text {bin }}^{\text {aniso }}=\left(\frac{m_{Q} \sigma^{2}}{m_{D}^{4} n^{2}}+\alpha m_{D}\right)+\frac{2 \xi}{3} \frac{m_{Q} \sigma^{2}}{m_{D}^{4} n^{2}},
$$

where the first term is the solution of (radial-part) of the Schrödinger equation with the isotropic part $\left(\operatorname{Re} V_{\text {iso }}(\hat{r} \gg 1, T)\right)$ and the second term is due to the anisotropic perturbation of the tensorial component $\left(V_{\text {tensor }}\left(\hat{r} \gg 1, \theta_{r}, T\right)\right)$ calculated from the first-order perturbation theory. The complex potential in general needs to be dealt with numerically to obtain the real and imaginary binding energies. Here we use the matrix method to solve the corresponding schrödinger equation (Thakur et al., 2013). 
Table 1: Dissociation temperatures of $J / \psi$ and $\Upsilon$ states for different anisotropies with the Debye mass in leading-order

\begin{tabular}{|c|c|c|c|c|}
\hline Method & State & $\xi=0.0$ & $\xi=0.3$ & $\xi=0.6$ \\
\hline \hline Re B.E.=Im B.E. & $J / \psi$ & 2.45 & 2.46 & 2.47 \\
& $\Upsilon$ & 3.40 & 3.45 & 3.46 \\
\hline Г=2B.E. & $J / \psi$ & 1.40 & 1.46 & 1.54 \\
& $\Upsilon$ & 3.10 & 3.17 & 3.26 \\
\hline
\end{tabular}
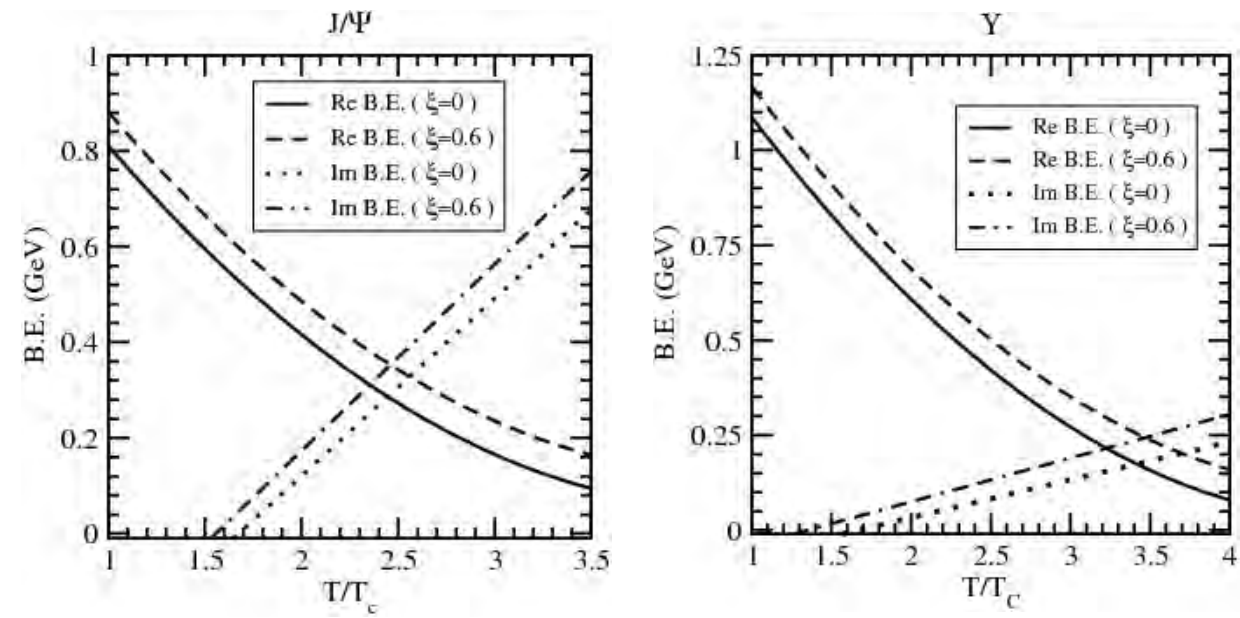

Fig. 3: Variation of the real and imaginary part of the binding energies for $J / \psi$ and $\Upsilon$ states for different anisotropies

We will now study the dissociation in thermal medium to calculate the dissociation temperature $\left(T_{d}\right)$ either from the intersection of the (real and imaginary) binding energies (Strickland and Bazow, 2012; Margotta et al., 2011) or from the conservative criterion on the width of the resonance as: $\Gamma \geq 2$ Re B.E. (Mocsy and Petreczky, 2008). Although both definitions are physically equivalent but they are numerically different (Table 1).

The real and imaginary part of the binding energies for the $J / \psi$ and $\Upsilon$ states are computed numerically in Fig. 3 for different values of anisotropies. We have computed the dissociation temperatures at different anisotropies in Table 1 from these numerical observations.

\section{Conclusion}

We have studied the properties of charmonium and bottomonium states with the in-medium modifications to both perturbative and non-perturbative part of the Cornell potential. The inclusion of the string term, in an(isotropic) medium, makes the quarkonium states more tight compared to the medium modification to the Coulomb term alone and increases the magnitude of the imaginary part. The presence of 
string terms broadens the (thermal) width of the states which plays an important role in the dissociation mechanism. We found that the quarkonium states are dissociated at higher temperature compared to the medium-consideration of the Coulomb term only. As the (effective) Debye mass in anisotropic medium is always smaller than that in isotropic medium, both the real and imaginary part of the potential becomes deeper with the increase of anisotropy and the binding of $Q \bar{Q}$ pairs becomes more stronger with respect to their isotropic counterpart. The overall observation is that the dissociation temperature increases with anisotropy and string term.

\section{Acknowledgement}

We are thankful for financial assistance from MHRD, India.

\section{References}

1. Agotiya V, Chandra V and Patra BK (2009) Dissociation of quarkonium in hot QCD medium: Modification of the inter-quark potential Phys Rev $\mathbf{C 8 0} 025210$

2. Brambilla N, Ghiglieri J, Vairo A and Petreczky P (2008) Static quark-antiquark pairs at finite temperature Phys Rev D78 014017

3. Beraudo A, Blaizot JP and Ratti C (2008) Real and imaginary-time Q anti-Q correlators in a thermal medium Nucl Phys A806 312-338

4. Burnier Y, Laine M and Vepsalainen M (2008) Heavy quarkonium in any channel in resummed hot QCD JHEP 01043

5. Carrington ME, Defu H and Thoma MH (1999) Equilibrium and nonequilibrium hard thermal loop resummation in the real time formalism Eur Phys $J$ C7 347-354

6. Cheng M, Datta S, Heide van der J, Huebner K et al. (2008) The Spatial String Tension and Dimensional Reduction in QCD Phys Rev D78 034506

7. Dumitru A, Guo Y and Strickland M (2009) The Imaginary part of the static gluon propagator in an anisotropic (viscous) QCD plasma Phys Rev D79 114003

8. Dumitru A (2011) Quarkonium in a non-ideal hot QCD Plasma Prog Theor Phys Suppl 18787

9. Hatsuda T (2013) Heavy quarkonium in hot medium Nucl Phys A904-905 210c-216c

10. Kapusta JI and Gale C (1996) Finite Temperature Field Theory Principle and Applications (Cambridge University Press, Cambridge) 2nd ed

11. Laine M, Philipsen O, Romatschke P and Tassler M (2007) Real-time static potential in hot QCD JHEP 03054

12. Laine M, Philipsen O, and Tassler M (2007) Thermal imaginary part of a real-time static potential from classical lattice gauge theory simulations JHEP 09066

13. Matsui T and Satz H (1986) $J / \psi$ suppression by quark-gluon plasma formation Phys Lett B178 416

14. Mocsy A and Petreczky P (2007) Color screening melts quarkonium Phys Rev Lett 99211602 
15. Mocsy A and Petreczky P (2008) Can quarkonia survive deconfinement? Phys Rev D77 014501

16. Mocsy A (2009) Potential Models for Quarkonia Eur Phys J C61 705-710

17. Megias E, Arriola ER and Salceedo LL(2007) The Quark-antiquark potential at finite temperature and the dimension two gluon condensate Phys Rev D75 105019

18. Megias E, Arriola ER and Salceedo LL (2011) Trace Anomaly and dimension two gluon condensate above the phase transition Indian J Phys 85 1191-1196

19. Margotta M, McCarty K, McGahan C, Strickland M and Elorriaga DY (2011) Quarkonium states in a complexvalued potential Phys Rev D83 105019

20. Romatschke P and Strickland M (2003) Collective modes of an anisotropic quark gluon plasma Phys Rev D68 036004

21. Rothkopf A, Hatsuda T and Sasaki S (2009) Proper heavy-quark potential from a spectral decomposition of the thermal Wilson loop PoS LAT 162

22. Rothkopf A, Hatsuda T and Sasaki S (2012) Complex Heavy-Quark Potential at Finite Temperature from Lattice QCD Phys Rev Lett 108162001

23. Schneider RA (2002) Debye screening at finite temperature, revisited Phys Rev $\mathbf{D 6 6} 036003$

24. Strickland M and Bazow D (2012) Thermal Bottomonium Suppression at RHIC and LHC Nucl Phys A879 25

25. Thakur L, Haque N, Kakade U and Patra BK (2013) Dissociation of quarkonium in an anisotropic hot QCD medium Phys Rev D88 054022. 